\title{
Integrable Evolution Equations on Associative Algebras
}

Peter J. Olver ${ }^{\dagger}$

School of Mathematics

University of Minnesota

Minneapolis, MN 55455

U.S.A.

olver@ima .umn . edu

http://ww . math . umn . edu/ olver
Vladimir V. Sokolov

Ufa Mathematical Institute

Russian Academy of Sciences

Chernyshevski str. 112

450000, Ufa

RUSSIA

sokolov@imat.rb.ru

\begin{abstract}
This paper surveys the classification of integrable evolution equations whose field variables take values in an associative algebra, which includes matrix, Clifford, and group algebra valued systems. A variety of new examples of integrable systems possessing higher order symmetries are presented. Symmetry reductions lead to associative algebra-valued version of the Painlevé transcendent equations. The basic theory of Hamiltonian structures for associative algebra-valued systems is developed and the biHamiltonian structures for several examples are found.
\end{abstract}

$\dagger$ Supported in part by NSF Grant DMS 95-00931.

$\$$ Supported in part by the Russian Foundation for Fundamental Research, Grant \#96-01-00382 and INTAS Grant \#93-166-EXT. 


\section{Introduction.}

This paper is devoted to the classification of certain non-commutative generalizations of classical integrable soliton equations. In the literature, integrable multi-component equations have been considered by Svinolupov, [48], [49], [51], [50], Fordy, [17], [18], and Marchenko, [29], and many other scientists. Svinolupov started the systematic classification of such systems, and observed that many arise from certain special types of nonassociative structures, such as Jordan algebras, Jordan triple systems, and so on. This approach leads to many new examples of integrable matrix and vector equations, [45], $[47]$, which are becoming the focus of significant research activity, [19].

Our starting point is the fact that most of the interesting examples appearing in these papers arise when the field variables take their value in an associative algebra. Particularly important cases are matrix or operator algebras, Clifford algebras, including the algebra of quaternions, and group algebras. Indeed, since the field variables can be regarded as taking values in an operator algebra, our classification approach can be considered as a constructive procedure for quantizing classical integrable systems. The associative algebra systems are the simplest, meaning that the algebraic theory and the computations are most easily understood. Svinolupov investigated integrable equations associated with special kinds of non-associative algebras, although he did not consider integrable equations arising from with the simpler associative algebras in any depth.

The existence of higher order symmetries has been effectively used to classify integrable evolution equations with commutative field variables, $[\mathbf{3 6}],[\mathbf{5 3}],[\mathbf{1 6}],[\mathbf{3 8}]$. Higher order symmetries typically occur in infinite hierarchies, obtained by successively applying a recursion operator to a trivial "seed" symmetry. (Bakirov, [5], proposes an example of a fourth order system with a single sixth order symmetry and no higher symmetries of order $\leq 60$; however, to date, no-one has been able to rigorously prove that Bakirov's system has no additional higher order symmetries.) Ibragimov, Shabat, Sokolov, and Mikhailov, $[\mathbf{2 2}],[31],[44]$, introduced the concept of a formal symmetry. Their method was then successfully used to classify several basic types of integrable evolutionary systems. We further refer the reader to the extensive tables in $[\mathbf{3 1}],[\mathbf{3 2}],[\mathbf{3 3}]$, for a summary of known examples. Due to the complexity of the computation, some of these classification tables relied on the existence of higher order conserved densities, and hence may not be complete. Indeed, as we demonstrate in our analysis of two-component systems of nonlinear Schrödinger (NLS) type, there are two integrable commutative two-component systems of NLS type which do not admit conserved densities and thus do not appear in the tables.

In this paper, we develop the symmetry classification of integrable systems arising from associative algebras in a systematic fashion. We have been able to find quite a few new and interesting examples of integrable systems using these methods. Many of our examples do not come from Jordan algebras, and thus lie outside the class of equations considered by Svinolupov, [51]. To make the classification procedure tractable, it is important that the dependent variable is not viewed as a matrix or vector with entries that can be combined arbitrarily, but only appears using intrinsic algebra operations of multiplication, addition, scalar multiplication, and, possibly, trace and transpose. One of our main points is that the higher order symmetries of the integrable matrix equations should themselves be local matrix equations. Although this is perhaps not surprising, it is not obvious from the work 
of Svinolupov and Fordy. Although we are as yet unable to conclusively demonstrate the locality of all the higher order symmetries, all known examples admit at least one higher order local matrix symmetry, and we choose this property to classify the equations.

A key question is how many of the known commutative examples extend to noncommutative integrable equations. We now list some known examples of integrable equations in which the field variable takes its values in an associative algebra. (For simplicity, the reader can view $u$ as a matrix-valued function.) The simplest case is, of course, a linear equation:

$$
u_{t}=u_{n}=D_{x}^{n}(u)
$$

The matrix-valued Burgers' equation comes in a right and left handed version,

$$
u_{t}=u_{x x}+u u_{x}, \quad \text { or } \quad u_{t}=u_{x x}+u_{x} u \text {. }
$$

Note that reversing the order of multiplication (or, in the matrix case, taking the transpose $\left.u \mapsto u^{*}\right)$ interchanges the two versions of Burgers' equation. The matrix Korteweg-deVries equation

$$
u_{t}=u_{x x x}+3 u u_{x}+3 u_{x} u=u_{x x x}+6\left\{u, u_{x}\right\}
$$

is invariant under transpose. Here $\{u, v\}=\frac{1}{2}(u v+v u)$ is the standard anti-commutator between fields $u$ and $v$. There are, remarkably, two different matrix versions of the modified Korteweg-deVries equation. The first is

$$
u_{t}=u_{x x x}+3 u^{2} u_{x}+3 u_{x} u^{2}=u_{x x x}+6\left\{u^{2}, u_{x}\right\}
$$

and is invariant under both $u \mapsto u^{*}$ and $u \mapsto-u$. The matrix Miura map

$$
u= \pm v_{x}+v^{2}
$$

maps this $\mathrm{mKdV}$ equation (for $v$ ) to the matrix $\mathrm{KdV}$ equation (1.3) for $u$. The second version is

$$
u_{t}=u_{x x x}+3 u u_{x x}-3 u_{x x} u-6 u u_{x} u=u_{x x x}+3\left[u, u_{x x}\right]-6 u u_{x} u
$$

which is invariant under only $u \mapsto-u^{*}$. This equation was first described in [26], where the Lax pair formulation and the inverse scattering problem were studied. Equation (1.6) does not admit a Miura transformation. Finally, the nonlinear Schrödinger equation has a noncommutative version

$$
u_{t}=u_{x x}+2 u v u, \quad v_{t}=-v_{x x}-2 v u v
$$

which is invariant under simultaneous transposes $u \mapsto u^{*}, v \mapsto v^{*}$. The system (1.7) can be identified with the usual (noncommutative) form

$$
\psi_{t}=i \psi_{x x}+2 \psi \bar{\psi} \psi
$$


of the nonlinear Schrödinger equation if we identify $u=\psi, v=\bar{\psi}$ and perform a complex scaling of the $x$ coordinate. Such complex transformations do not affect the algebraic integrability of the equation, but do, of course, have ssignificant effects on its analytical properties and (real) solutions.

Not every scalar integrable system has a noncommutative counterpart. There are no purely matrix analogues of the fifth order Sawada-Kotera, [42], and Kaup, [25], equations when the right hand side of the evolution equation only involves the field variable $u$ and its derivatives, but we suspect there may be a form that also depends on the transpose $u^{*}$ and its derivatives. However, the complexity of the calculations have so far prevented us for determining the precise forms of the desired equations.

Symmetry reductions of classical soliton equations lead to ordinary differential equations of Painlevé type, meaning those whose movable singularities are only poles, [2]. Analogous reductions of our noncommutative integrable systems will therefore lead to new associative algebra-valued ordinary differential equations of Painlevé type. In particular, we exhibit analogues of some of the classical Painlevé transcendents. However, it remains to determine the types of singularities and perform a Painlevé classification of such systems in a direct fashion. We refer the reader to $[\mathbf{4 1}],[\mathbf{5 2}]$, for additional applications of associative, Jordan, and other types of algebras to ordinary differential equations.

A particularly powerful approach to integrability was discovered by Magri, [28], who showed how equations possessing two distinct compatible Hamiltonian structures have a recursion operator, and corresponding infinite hierarchy of commuting biHamiltonian flows and associated conservation laws. As in the commutative case, some of the integrable equations over associative algebras, notably the analogues of the $\mathrm{KdV}, \mathrm{mKdV}$, and nonlinear Schrödinger equations are also biHamiltonian systems. In order to understand this additional structure, we shall need to develop the theory of functionals and Hamiltonian operators over associative algebras having an additional trace operation. The verification of the all-important Jacobi identity requires us to develop the associated theory of noncommutative functional multi-vectors, in direct analogy with the commutative version developed in [38; Chapter 7]. The biHamiltonian approach provides a simplified proof of the existence of suitable recursion operators.

Remarkably, one consequence of our studies is that the first order Hamiltonian operators associated with systems of hydrodynamic type, as considered by Dubrovin and Novikov, [12], [13], [14], do not naturally generalize to local Hamiltonian operators in noncommutative variables: one is required to append certain nonlocal terms in order to satisfy the Jacobi identity. The resulting noncommutative operators have some similarities with the non-local (but commutative) Hamiltonian operators of hydrodynamic type introduced by Mokhov, Ferapontov, [15], [34], [35]. Given the beautiful and deep connections between such Hamiltonian operators and classical Riemannian geometry, this result invites an interesting speculation on the proper form of a noncommutative Riemannian geometry, [8], that will produce such nonlocal Hamiltonian operators.

We begin our paper with a review of the basic facts from the theory of associative algebras. In section 3 we present classification results for one-component evolution equations, of orders 2, 3, and 5. Section 4 discusses the classification of two-component systems of nonlinear Schrödinger type. Section 5 provides a brief discussion of the theory of non- 
commutative Painlevé equations. The last section develops the noncommutative theory of Hamiltonian and biHamiltonian systems, with emphasis on the KdV and mKdV systems.

\section{Associative Algebras.}

In this paper, the field variables in our systems will take their values in a linear associative algebra $\mathcal{A}$. Three important examples are the algebras of $n \times n$ matrices, Clifford algebras, and the group algebras appearing in the representation theory of finitedimensional groups. Our results, though, do not depend on $\mathcal{A}$ being finite-dimensional, and so we could also view $\mathcal{A}$ as a suitable operator algebra over, say, Hilbert space.

For brevity, the noncommutative multiplication $u v$ for $u, v \in \mathcal{A}$ will be just denoted by juxtaposition. We use the notation

$$
L_{u}(v)=u v, \quad R_{u}(v)=v u,
$$

for the operators of left and right multiplication. The commutator and anti-commutator on $\mathcal{A}$ are denoted by the standard bracket notations

$$
[u, v]=u v-v u, \quad\{u, v\}=\frac{1}{2}(u v+v u)
$$

We also introduce the notation

$$
C_{u}(v)=[u, v], \quad A_{u}(v)=2\{u, v\}
$$

so that

$$
C_{u}=L_{u}-R_{u}, \quad A_{u}=L_{u}+R_{u} .
$$

Note that the anti-commutator defines a Jordan algebra structure on $\mathcal{A},[24]$. Finally, for notational convenience, we introduce the "triple anti-commutator"

$$
\{u, v, w\}=\frac{1}{2}(u v w+w v u)
$$

Note that $\{u, v\}=\{u, \mathbf{e}, v\}$ where $\mathbf{e}$ is the identity element of $\mathcal{A}$.

Each of our associative algebras comes with an involution $u \mapsto u^{*}$, called the transpose, that interchanges the operations of left and right multiplication, so $(u v)^{*}=v^{*} u^{*}$. In certain instances, we will also require the existence of a trace form on the algebra, by which we mean a scalar-valued invariant multilinear form $\operatorname{tr}: \mathcal{A} \times \mathcal{A} \rightarrow \mathbb{R}$, that satisfies

$$
\operatorname{tr} u v=\operatorname{tr} v u, \quad \text { so that } \quad \operatorname{tr}[u, v]=0 .
$$

The simplest example of an associative algebra is, as mentioned above, the algebra of real $n \times n$ matrices, which we denote by $\mathfrak{g l}(n, \mathbb{R})$. More generally, if $\mathcal{A}$ is any associative algebra, then the space $\mathfrak{g l}(n, \mathcal{A})$ of $n \times n$ matrices whose entries belong to $\mathcal{A}$ also forms an associative algebra. Since $\mathfrak{g l}(m, \mathfrak{g l}(n, \mathcal{A})) \simeq \mathfrak{g l}(m n, \mathcal{A})$, iterating this procedure does not produce anything new. Similarly, one can form "vector" associative algebras by taking the 
direct sum of two or more associative algebras, e.g., $\mathcal{A} \oplus \mathcal{B}$, and using the component-wise multiplication $(a, b) \cdot(c, d)=(a c, b d)$.

Clifford algebras form a second important class of associative algebras, cf. [40]. The simplest non-commutative Clifford algebra is the algebra $\mathbb{H}$ of quaternions. We write $u=v+\mathbf{w}$ where $v \in \mathbb{R}$ is the scalar and $\mathbf{w} \in \mathbb{R}^{3}$ the vector part of $u$. Note that the associative quaternion multiplication is

$$
u \hat{u}=(v \hat{v}-\mathbf{w} \cdot \widehat{\mathbf{w}})+v \widehat{\mathbf{w}}+\hat{v} \mathbf{w}+\mathbf{w} \times \widehat{\mathbf{w}},
$$

where $\cdot$ is the ordinary dot product and $\times$ the ordinary cross product in three-dimensional space. The general classification of Clifford algebras can be found, for instance, in [40].

Theorem 2.1. Every Clifford algebra over $\mathbb{R}$ is isomorphic to one of the following matrix algebras

$$
\mathfrak{g l}\left(2^{k}, \mathbb{R}\right), \quad \mathfrak{g l}\left(2^{k}, \mathbb{C}\right), \quad \mathfrak{g l}\left(2^{k}, \mathbb{H}\right), \quad \mathfrak{g l}\left(2^{k}, \mathbb{R} \oplus \mathbb{R}\right), \quad \mathfrak{g l}\left(2^{k}, \mathbb{H} \oplus \mathbb{H}\right) .
$$

Therefore, every Clifford algebra is constructed as a matrix algebra (of a particular size) over the basic algebras $\mathbb{R}, \mathbb{C}, \mathbb{H}$, and the two vector algebras $\mathbb{R} \oplus \mathbb{R}$, and $\mathbb{H} \oplus \mathbb{H}$.

If $G$ is a finite group containing $r$ elements, then the (real) group algebra $\mathcal{A}_{G}=\mathbb{R}[G]$ is an $r$-dimensional vector space, whose basis elements are identified with the elements of $G$. Thus, the elements of $\mathbb{R}[G]$ have the form $u=\sum_{g \in G} u^{g} \cdot g$, with coefficients $u^{g} \in \mathbb{R}$ indexed by the group elements. The group algebra multiplication is induced from that of the group itself. Thus, if $w=u v$, then

$$
w^{g}=\sum_{h \in G} u^{h} v^{h^{-1} g}
$$

where $h^{-1} g$ denotes the multiplication of elements $h, g \in G$. Note that the group algebra is commutative if and only if $G$ is abelian. Thus, the simplest noncommutative group algebra is the six-dimensional algebra $\mathbb{R}\left[\mathbf{S}^{3}\right]$ associated with the symmetric group on three letters.

\section{Classification of One-Component Evolution Equations.}

Let $\mathcal{A}$ be an associative algebra. We can use the linear structure on $\mathcal{A}$ to differentiate maps $u: \mathbb{R} \rightarrow \mathcal{A}$, leading to an algebra of associative, but not necessarily commutative differential polynomials, which we denote by $\mathcal{D}=\mathcal{D}[x, u]$. We use subscript notation for derivatives, so that $u_{x}, u_{x x}$, etc. denote the first, second, etc. derivatives of $u=u(x)$ with respect to to its argument $x$. For simplicity, we only consider $x$-independent differential polynomials in this paper, although the general methods readily extend to differential polynomials that have $x$-dependent coefficients.

A one-component ${ }^{\dagger} \mathcal{A}$-valued evolution equation will then be of the general form

$$
u_{t}=K[u], \quad \text { where } \quad K \in \mathcal{D} \text {. }
$$

$\dagger$ In the commutative case, a one-component equation is a scalar equation, but the use of the term "scalar" here would be confusing. 
In the following section, we consider multi-component equations. Each evolution equation describes the flow associated with an evolutionary vector field

$$
\mathbf{v}_{K}=\sum_{n=1}^{\infty} D_{x}^{n} K \frac{\partial}{\partial u_{n}},
$$

which acts on the space of differential polynomials, cf. [38]. Two such equations are called equivalent if they can be mapped to each other by a scaling transformation

$$
(x, t, u) \longmapsto(\lambda x, \mu t, \nu u) .
$$

The evolution equation (3.1) is said to be integrable if it admits a higher order differential polynomial symmetry, again described by an evolution equation

$$
u_{t}=S[u] \text {. }
$$

The symmetry condition that the two evolutionary flows (3.1), (3.4) commute implies that the two evolutionary vector fields commute: $\left[\mathbf{v}_{K}, \mathbf{v}_{S}\right]=0$. Define the Fréchet derivative of the differential polynomial $K$ by adapting the basic formula

$$
D_{K}[w]=\left.\frac{d}{d \varepsilon} K[u+\varepsilon w]\right|_{\varepsilon=0},
$$

from the commutative case, $\mathrm{cf}$. $[\mathbf{3 8}]$. Since $\mathbf{v}_{K}[S]=D_{S}[K]$, the commutativity of the flows (3.1), (3.4), can then be written in the usual form

$$
D_{K}[S]-D_{S}[K]=0 .
$$

Given an evolution equation (3.1), the determination of all symmetries (3.4) of a given order $m$ is a straightforward, but computationally intensive, calculation. We have implemented a MATHEMATICA package which will automatically do these computations. The programs for effecting such computations are available at the web site

$$
\text { http : //www.math.umn.edu/ olver. }
$$

Even so, due to time and memory resources, only a limited number of such computations can be completed.

In all interesting examples, the right hand side of an integrable evolution equation (3.1) turns out to be a homogeneous differential polynomial with respect to some weighting of its constituent monomials. We introduce a weighting scheme on the algebra $\mathcal{D}$ by assigning a weight $m=\operatorname{deg} u$ to the dependent variable and $n=\operatorname{deg} x$ to the independent variable, so that the $k^{\text {th }}$ order derivative of $u$ with respect to $x$ has weight $m+k n$. We shall, without loss of generality, assume that $\operatorname{deg} x=1$. The weight of a monomial is the sum of the weights of its multiplicands. We let $\mathcal{D}^{(n)}$ denote the space of differential polynomials of weight $n$. For example, the $\mathrm{KdV}$ weighting has

$$
\operatorname{deg} u=2, \quad \operatorname{deg} x=1 .
$$

In the noncommutative case, there are three monomials of weight 5 , namely $u_{x x x}, u u_{x}$ and $u_{x} u$. Every differential polynomial $P \in \mathcal{D}^{(5)}$ of weight 5 is thus a linear combination of these three monomials. Clearly, if (3.1) is a homogeneous evolution equation, then the homogeneous summands of any symmetry are automatically symmetries, and hence we can, without loss of generality, restrict our attention to symmetries $S \in \mathcal{D}^{(m)}$ that are homogeneous in the prescribed weighting. 
Definition 3.1. Under a given weighting scheme, the weight of an evolution equation (3.1) is equal to the difference $\operatorname{deg} K-\operatorname{deg} u$.

For example, under the KdV weighting (3.7), the most general evolution equation of weight 3 has the form

$$
u_{t}=a u_{x x x}+b u u_{x}+c u_{x} u
$$

where $a, b, c \in \mathbb{R}$. The reason for the definition of weighting is so that, when $x$ has degree 1 , the linear terms in an evolution equation of weight $k$ are of order $k$, and so, in most cases, the weight of the evolution equation equals its order. More specifically:

Definition 3.2. A homogeneous evolution equation is nondegenerate if it contains a linear term.

Lemma 3.3. If $\operatorname{deg} x=1$, then a nondegenerate homogeneous evolution equation of weight $k$ is a $k^{\text {th }}$ order differential equation.

As a consequence of Definition 3.1, the commutator equation $u_{t}=D_{K}[S]-D_{S}[K]$ of a weight $k$ equation and weight $l$ symmetry has weight $k+l$. (However, in the one component case, it is never nondegenerate!)

We now summarize the results obtained to date through the symmetry classification of nondegenerate homogeneous polynomial evolution equations (3.1). The following results are the consequences of extensive computer algebra computations using our MATHEMATICA package.

Theorem 3.4. For the $K d V$ weighting (3.7), every nondegenerate polynomial equation of weight 3 over an associative algebra having a weight 5 symmetry is either linear or equivalent to the Korteweg-deVries equation (1.3).

For example, the fifth order symmetry of the Korteweg-deVries equation (1.3) is

$$
u_{t}=u_{x x x x x}+10\left\{u, u_{x x x}\right\}+20\left\{u_{x}, u_{x x}\right\}+20\left\{u^{2}, u_{x}\right\}+10 u u_{x} u
$$

Note that, in the commutative case, this reduces to the standard fifth order symmetry of the ordinary Korteweg-deVries equation, [38].

In the commutative case, there are three different fifth order integrable polynomial equations, $[\mathbf{3 1}],[\mathbf{2 7}]$; besides the fifth order $\mathrm{KdV}$ equation, these are the Sawada-Kotera equation, $[42]$, and the Kaup-Kupershmidt equation, $[\mathbf{2 5}]$. We have shown that neither of these has an associative counterpart where the right hand side is a differential polynomial in $u$ alone, although we believe that there do exist integrable versions involving $u$ and its transpose $u^{*}$.

Theorem 3.5. For the $K d V$ weighting, every nondegenerate polynomial equation of weight 5 over an associative algebra having a weight 7 symmetry is either linear or equivalent to the fifth order Korteweg-deVries equation (3.9). 
Using the triple bracket notation (2.4), we can write the seventh order symmetry of the fifth order Korteweg-deVries equation (1.3) in the form

$$
\begin{aligned}
& u_{t}=u_{x x x x x x}+14\left\{u, u_{x x x x x}\right\}+42\left\{u_{x}, u_{x x x x}\right\}+70\left\{u_{x x}, u_{x x x}\right\}+ \\
& +42\left\{u^{2}, u_{x x x}\right\}+28 u u_{x x x} u+98\left\{u, u_{x}, u_{x x}\right\}+112\left\{u, u_{x x}, u_{x}\right\}+ \\
& +70\left\{u_{x}, u, u_{x x}\right\}+70 u_{x}^{3}+70\left\{u^{3}, u_{x}\right\}+70\left\{u^{2}, u_{x}, u\right\} .
\end{aligned}
$$

The second interesting weighting is that associated with both Burgers' equation and the modified Korteweg-deVries ( $\mathrm{mKdV}$ ) equation:

$$
\operatorname{deg} u=1, \quad \operatorname{deg} x=1 .
$$

Theorem 3.6. For the equal weighting (3.11), every nondegenerate polynomial equation of weight 2 over an associative algebra having a weight 3 symmetry is either linear or equivalent to either the right or left handed Burgers' equations (1.2). Every polynomial equation of weight 3 having a weight 5 symmetry is either linear or equivalent to one of the following five equations:

$$
\begin{aligned}
& u_{t}=u_{x x x}+6\left\{u^{2}, u_{x}\right\}, \\
& u_{t}=u_{x x x}+3\left[u, u_{x x}\right]-6 u u_{x} u, \\
& u_{t}=u_{x x x}+3 u_{x}^{2}, \\
& u_{t}=u_{x x x}+3 u u_{x x}+3 u_{x}^{2}+3 u^{2} u_{x}, \\
& u_{t}=u_{x x x}+3 u_{x x} u+3 u_{x}^{2}+3 u_{x} u^{2} .
\end{aligned}
$$

The first and second of these integrable equations are the two versions of the $\mathrm{mKdV}$ equation discussed above. The third is the potential $\mathrm{KdV}$ equation. The fourth and fifth are the third order symmetries of the right and left handed Burgers' equations (1.2), respectively - these systems also admit fourth order symmetries.

Each of the preceding one-component noncommutative integrable equations can be made into an integrable system by identifying the dependent variable as a matrix-valued function $u(x, t)=\left(u_{\beta}^{\alpha}(x, t)\right)$. A second interesting class of systems comes from the case when $u=v+\mathbf{w} \in \mathbb{H}$ takes its values in the quaternions, where $v \in \mathbb{R}, \mathbf{w} \in \mathbb{R}^{3}$, as in (2.6). The quaternion versions of our integrable equations are as follows:

(1) Quaternion Burgers' equation:

$$
\begin{aligned}
v_{t} & =v_{x x}+v v_{x}-\mathbf{w} \cdot \mathbf{w}_{x} \\
\mathbf{w}_{t} & =\mathbf{w}_{x x}+(v \mathbf{w})_{x}+\mathbf{w} \times \mathbf{w}_{x} .
\end{aligned}
$$


(2) Quaternion Korteweg-deVries equation:

$$
\begin{aligned}
v_{t} & =v_{x x x}+3\left(v^{2}-|\mathbf{w}|^{2}\right)_{x}, \\
\mathbf{w}_{t} & =\mathbf{w}_{x x x}+6(v \mathbf{w})_{x}
\end{aligned}
$$

This coincides with the well-known vector $\mathrm{KdV}$ equation given in [45].

(3) The first quaternion modified Korteweg-deVries equation is

$$
\begin{aligned}
v_{t} & =v_{x x x}+\left(2 v^{3}-6 v|\mathbf{w}|^{2}\right)_{x}, \\
\mathbf{w}_{t} & =\mathbf{w}_{x x x}+6\left(v^{2} \mathbf{w}\right)_{x}-6|\mathbf{w}|^{2} \mathbf{w}_{x} .
\end{aligned}
$$

This is also a well-known vector $\mathrm{mKdV}$ equation.

(4) The second quaternion $\mathrm{mKdV}$ is more interesting:

$$
\begin{aligned}
v_{t} & =v_{x x x}+\left(6|\mathbf{w}|^{2} v-2 v^{3}\right)_{x}, \\
\mathbf{w}_{t} & =\mathbf{w}_{x x x}+6\left(\mathbf{w} \times \mathbf{w}_{x}-\left(v^{2}+|\mathbf{w}|^{2}\right) \mathbf{w}\right)_{x}+18|\mathbf{w}|^{2} \mathbf{w}_{x} .
\end{aligned}
$$

Note that the reduction $v=0$ leads to the three-dimensional vector equation

$$
\mathbf{w}_{t}=\mathbf{w}_{x x x}+6 \mathbf{w} \times \mathbf{w}_{x x}-6\left(\mathbf{w} \cdot \mathbf{w}_{x}\right) \mathbf{w}+6|\mathbf{w}|^{2} \mathbf{w}_{x} .
$$

Finally, we can also consider cases in which the field variable $u$ takes its values in a group algebra $\mathbb{R}[G]$. The multiplication rule (2.8) allows us to write out the component form of the equation. For example, the Korteweg-deVries equation (1.3) on a group algebra has the component form

$$
u_{t}^{g}=u_{x x x}^{g}+3 \sum_{h \in G}\left[u^{h^{-1} g}+u^{g h^{-1}}\right] u_{x}^{h}, \quad g \in G .
$$

The indices in (3.18) are governed by the group multiplication. The G-KdV system (3.18) is integrable for any finite group $G$. In particular, if $G$ is abelian, then the two summands in (3.18) coincide.

Because of the variety of isomorphisms between matrix, Clifford, and group algebras, our three classes of associative algebras very often lead to different forms of the same equation. For example, in view of Theorem 2.1, the associative equations on higher dimensional Clifford algebras are straightforward matrix versions of either the real, the complex, or the quaternionic equations. The vector integrable systems in $[45]$ arise from Clifford algebras. The evolution equations associated with the last two matrix Clifford algebras $\mathbb{R} \oplus \mathbb{R}$ and $\mathbb{H} \oplus \mathbb{H}$ automatically decouple into two independent evolution equations associated with the real or quaternionic matrix algebra, and are thus not particularly interesting. 


\section{Integrable Two-Component Systems on Associative Algebras.}

The classification of associative algebra valued systems having two or more field variables is even less well developed. We shall only consider second order systems of the form

$$
\mathbf{u}_{t}=A \mathbf{u}_{x x}+\mathbf{F}\left(\mathbf{u}, \mathbf{u}_{x}\right)
$$

where $\mathbf{u}=\left(u^{1}, \ldots, u^{n}\right)$ and $A$ is a constant $n \times n$ matrix. Note that, by a (complex) linear transformation $\mathbf{u} \mapsto B \mathbf{u}$, we can place $A$ into Jordan canonical form. Moreover, scaling $x$ will allow us to make at least one of the diagonal entries of $A$ equal to 1 .

Mikhailov, Shabat and Yamilov, [32], [33], investigated the two component systems, $n=2$, in detail, and used the existence of higher order conservation laws to characterize integrability. Here we continue their work in both the commutative and noncommutative cases. For simplicity, we assume that the linear part of the system (4.1) is prescribed by the simple diagonal matrix $A=\operatorname{diag}(1,-1)$, leaving other types of two component systems to future investigations. We will consider a second order system to be integrable if it possesses a fourth order symmetry. All known examples of integrable second order systems satisfy this condition.

Definition 4.1. A two component system is called triangular if it decouples into the form

$$
u_{t}=F\left(u, u_{x}, u_{x x}\right), \quad v_{t}=G\left(u, v, u_{x}, v_{x}, u_{x x}, v_{x x}\right)
$$

Note that a triangular system can be solved by first solving the one-component equation for $u$ and then using that to reduce the $v$ equation to a second $x, t$ dependent onecomponent equation. In our classification procedure, for brevity we have chosen not to consider systems which are equivalent, under a point transformation, to triangular systems, so we can concentrate on "genuinely" two-component systems.

The first class of systems are the noncommutative versions of the nonlinear Schrödinger equation, which corresponds to the weighting

$$
\operatorname{deg} u=1, \quad \operatorname{deg} v=1, \quad \operatorname{deg} x=1 .
$$

The general form of such a second order system in the commutative case is

$$
\begin{aligned}
& u_{t}=u_{x x}+a_{1} u u_{x}+a_{2} v u_{x}+a_{3} u v_{x}+a_{4} v v_{x}+b_{1} u^{3}+b_{2} u^{2} v+b_{3} u v^{2}+b_{4} v^{3} \\
& v_{t}=-v_{x x}-c_{4} u u_{x}-c_{3} v u_{x}-c_{2} u v_{x}-c_{1} v v_{x}-d_{4} u^{3}-d_{3} u^{2} v-d_{2} u v^{2}-d_{1} v^{3}
\end{aligned}
$$

The corresponding fourth order symmetry has the form

$$
\begin{aligned}
& u_{\tau}=u_{x x x x}+f\left(u, v, u_{x}, v_{x}, u_{x x}, v_{x x}, u_{x x x}, v_{x x x}\right) \\
& v_{\tau}=-v_{x x x x}+g\left(u, v, u_{x}, v_{x}, u_{x x}, v_{x x}, u_{x x x}, v_{x x x}\right) .
\end{aligned}
$$

All known integrable commutative equations (4.4) have a polynomial symmetry of the form (4.5). 
Theorem 4.2. Up to scaling of $t, x, u, v$, and the discrete transformation

$$
u \longleftrightarrow v, \quad t \longleftrightarrow-t,
$$

every nonlinear, commutative nontriangular system of the form (4.4), possessing a polynomial symmetry of the form (4.5) is equivalent to one of the following seven integrable systems:

$$
\begin{aligned}
& \left\{\begin{array}{l}
u_{t}=u_{x x}+2 u^{2} v \\
v_{t}=-v_{x x}-2 v^{2} u,
\end{array}\right. \\
& \left\{\begin{array}{l}
u_{t}=u_{x x}+2 v v_{x} \\
v_{t}=-v_{x x}+2 u u_{x}
\end{array}\right. \\
& \left\{\begin{array}{l}
u_{t}=u_{x x}+2 u u_{x}+2 v u_{x}, \\
v_{t}=-v_{x x}+2 u v_{x}+2 v v_{x}
\end{array},\right. \\
& \left\{\begin{array}{l}
u_{t}=u_{x x}+2 u u_{x}+2 v u_{x}+2 u v_{x}, \\
v_{t}=-v_{x x}+2 v u_{x}+2 u v_{x}+2 v v_{x}
\end{array},\right. \\
& \left\{\begin{array}{l}
u_{t}=u_{x x}+2 u v_{x}+2 v v_{x}-\frac{2}{3}(u+v)^{3}, \\
v_{t}=-v_{x x}+2 u u_{x}+2 v u_{x}+\frac{2}{3}(u+v)^{3},
\end{array}\right. \\
& \left\{\begin{array}{l}
u_{t}=u_{x x}-2 u u_{x}-2 v u_{x}-2 u v_{x}+2 u^{2} v+2 u v^{2}, \\
v_{t}=-v_{x x}+2 v u_{x}+2 u v_{x}+2 v v_{x}-2 u^{2} v-2 u v^{2},
\end{array}\right. \\
& \left\{\begin{array}{l}
u_{t}=u_{x x}+2 u u_{x}+2 v v_{x}, \\
v_{t}=-v_{x x}-v u_{x}-u v_{x}-\frac{3}{4} u^{2} v-\frac{1}{2} v^{3},
\end{array}\right.
\end{aligned}
$$

The last two systems do not appear in the lists of Mikhailov, Shabat and Yamilov, $[\mathbf{3 2}],[33]$, because they do not have higher order conserved densities. System (4.12) was first obtained by A.E. Borovik, V.Yu. Popkov and V.N. Robuk, cf. [43; (4.5)], and can be linearized by a differential substitution. System (4.13) looks new.

In the noncommutative case, we are classifying systems of the general form

$$
\begin{gathered}
u_{t}=u_{x x}+a_{1} u u_{x}+a_{2} u v_{x}+a_{3} v u_{x}+a_{4} v v_{x}+a_{5} u_{x} u+a_{6} u_{x} v+a_{7} v_{x} u+a_{8} v_{x} v+ \\
+b_{1} u^{3}+b_{2} u^{2} v+b_{3} u v u+b_{4} u v^{2}+b_{5} v u^{2}+b_{6} v u v+b_{7} v^{2} u+b_{8} v^{3} \\
v_{t}=-v_{x x}-c_{1} v v_{x}-c_{2} v u_{x}-c_{3} u v_{x}-c_{4} u u_{x}-c_{5} v_{x} v-c_{6} v_{x} u-c_{7} u_{x} v-c_{8} u_{x} u- \\
\quad-d_{1} v^{3}-d_{2} v^{2} u-d_{3} v u v-d_{4} v u^{2}-d_{5} u v^{2}-d_{6} u v u-d_{7} u^{2} v-d_{8} u^{3}
\end{gathered}
$$

up to equivalence, where we allow scaling, the discrete transformation (4.6), as well as the formal involution $(u, v) \mapsto\left(u^{*}, v^{*}\right)$ that interchanges the order of multiplication. 
The complete list of the nonlinear, nontriangular noncommutative systems up to equivalence is as follows. Interestingly, each of the first six commutative nontriangular integrable systems has either one or two noncommutative counterparts; however, the final new system does not generalize to the noncommutative regime. These integrable noncommutative systems naturally split into three classes. The first group contains the six systems

$$
\begin{aligned}
& \left\{\begin{array}{l}
u_{t}=u_{x x}+2 u v u \\
v_{t}=-v_{x x}-2 v u v,
\end{array}\right. \\
& \left\{\begin{array}{l}
u_{t}=u_{x x}+2 u u_{x}-2 u v_{x}+2 v u_{x}+2 v_{x} u-2 u u v+4 u v u-2 v u u, \\
v_{t}=-v_{x x}+2 v u_{x}-2 u_{x} v+2 v_{x} u+2 v_{x} v+2 u v v-4 v u v+2 v v u,
\end{array}\right. \\
& \left\{\begin{array}{l}
u_{t}=u_{x x}+2 u u_{x}+2 v u_{x} \\
v_{t}=-v_{x x}+2 v_{x} u+2 v_{x} v
\end{array}\right. \\
& \left\{\begin{array}{l}
u_{t}=u_{x x}+2 u u_{x}+2 v u_{x}+2 v_{x} u+2 u v u-2 v u^{2}, \\
v_{t}=-v_{x x}+2 v u_{x}+2 v_{x} u+2 v_{x} v-2 v u v+2 v^{2} u,
\end{array}\right. \\
& \left\{\begin{array}{l}
u_{t}=u_{x x}+2 u u_{x}+2 u v_{x}+2 u_{x} v+2 u^{2} v-2 u v u, \\
v_{t}=-v_{x x}+2 v u_{x}+2 v v_{x}+2 v_{x} u+2 v u v-2 v^{2} u,
\end{array}\right. \\
& \left\{\begin{array}{l}
u_{t}=u_{x x}+2 u u_{x}-2 v u_{x}-2 v_{x} u-2 u v u+2 v^{2} u, \\
v_{t}=-v_{x x}-2 u v_{x}+2 v v_{x}-2 u_{x} v-2 u^{2} v+2 v u v .
\end{array}\right.
\end{aligned}
$$

The commutative versions of these are the following: (4.15) is a noncommutative generalization of (4.7); both (4.16) and (4.17) reduce to (4.9); (4.18) and (4.19) reduce to (4.10), while $(4.20)$ reduces to $(4.12)$.

The second group involves a primitive cube root of unity $\epsilon$, where $\epsilon^{2}+\epsilon+1=0$. We can set

$$
\epsilon=\exp \frac{2 \pi i}{3}=-\frac{1}{2}+\frac{i \sqrt{3}}{2}
$$

without loss of generality. The following four systems lie in this class:

$$
\begin{aligned}
& \left\{\begin{array}{c}
u_{t}=u_{x x}-2 \epsilon v v_{x}+2 v_{x} v-2(1+\epsilon) u^{2} v+2 u v u+2 \epsilon v u^{2} \\
v_{t}=-v_{x x}+2(1+\epsilon) u u_{x}+2 u_{x} u+2(1+\epsilon) u v^{2}-2 v u v-2 \epsilon v^{2} u
\end{array}\right. \\
& \left\{\begin{array}{r}
u_{t}=u_{x x}+2 u u_{x}+2 u v_{x}-2 v u_{x}+2(1+\epsilon) v v_{x}-2 u_{x} u+2 u_{x} v-2 v_{x} u+2 \epsilon v_{x} v+ \\
\quad+2(1-\epsilon) u^{2} v-6 u v u+2(1+2 \epsilon) u v^{2}+2(2+\epsilon) v u^{2}-2(1+2 \epsilon) v^{2} u \\
v_{t}=-v_{x x}-2 \epsilon u u_{x}-2 u v_{x}+2 v u_{x}+2 v v_{x}-2(1+\epsilon) u_{x} u-2 u_{x} v+2 v_{x} u- \\
-2 v_{x} v-2(1+2 \epsilon) u^{2} v-2(1-\epsilon) u v^{2}+2(1+2 \epsilon) v u^{2}+6 v u v-2(2+\epsilon) v^{2} u
\end{array}\right.
\end{aligned}
$$




$$
\begin{gathered}
\left\{\begin{array}{r}
u_{t}=u_{x x}+2 u u_{x}-2 \epsilon u v_{x}-2 v u_{x}+2(1+\epsilon) v v_{x}-2 u_{x} u+2 u_{x} v- \\
-2(1+\epsilon) v_{x} u+2 \epsilon v_{x} v+2 u^{3}-2(1+2 \epsilon) u^{2} v-6 u v u+ \\
+4(1+\epsilon) u v^{2}+2(1+2 \epsilon) v u^{2}+2 v u v-4 \epsilon v^{2} u-2 v^{3}, \\
v_{t}=-v_{x x}-2 \epsilon u u_{x}-2 u v_{x}+2(1+\epsilon) v u_{x}+2 v v_{x}-2(1+\epsilon) u_{x} u+ \\
+2 \epsilon u_{x} v+2 v_{x} u-2 v_{x} v+2 u^{3}-4(1+\epsilon) u^{2} v-2 u v u+ \\
+2(1+2 \epsilon) u v^{2}+4 \epsilon v u^{2}+6 v u v-2(1+2 \epsilon) v^{2} u-2 v^{3},
\end{array}\right. \\
\left\{\begin{array}{r}
u_{t}=u_{x x}+2(\epsilon+1) u v_{x}+2(\epsilon+1) v v_{x}+2 \epsilon v_{x} u+2 \epsilon v_{x} v+ \\
+2 u^{3}+2 u^{2} v+2 u v u+2 u v^{2}+2 v u^{2}+2 v u v+2 v^{2} u+2 v^{3}, \\
v_{t}=-v_{x x}+2 \epsilon u u_{x}+2 \epsilon v u_{x}+2(\epsilon+1) u_{x} u+2(\epsilon+1) u_{x} v- \\
-2 u^{3}-2 u^{2} v-2 u v u-2 u v^{2}-2 v u^{2}-2 v u v-2 v^{2} u-2 v^{3} .
\end{array}\right.
\end{gathered}
$$

Both (4.21) and (4.22) reduce to (4.8), while (4.23) and (4.24) reduce to (4.11). The final noncommutative nontriangular equation is

$$
\left\{\begin{array}{r}
u_{t}=u_{x x}+2 u u_{x}-2 u v_{x}+2 v u_{x}-2 u_{x} v+2 v_{x} u- \\
-2 u u v+4 u v u+2 u v v-2 v u u-2 v u v \\
v_{t}=-v_{x x}+2 v u_{x}+2 v_{x} u+2 v_{x} v-2 v u v+2 v v u
\end{array}\right.
$$

This case reduces to a commutative triangular system

$$
u_{t}=u_{x x}+2 u u_{x}, \quad v_{t}=-v_{x x}+2 v u_{x}+2 v_{x} u+2 v_{x} v
$$

Theorem 4.3. Every associative algebra valued, two-component nontriangular second order system of nonlinear Schrödinger form (4.14) possessing a fourth order symmetry is equivalent to one of the systems (4.15-4.25).

Interestingly, most of these integrable systems are not associated with a Jordan algebra, and thus lies outside the class of equations considered by Svinolupov, [50], [51].

Next, we consider the weighting

$$
\operatorname{deg} u=1, \quad \operatorname{deg} v=1, \quad \operatorname{deg} x=2,
$$

which governs the derivative nonlinear Schrödinger equation. We have not performed a complete classification in this case. We did find two nontriangular integrable systems of second order with the given diagonal matrix as linear part:

$$
\begin{gathered}
\left\{\begin{array}{l}
u_{t}=u_{x x}+2(u v u)_{x}, \\
v_{t}=-v_{x x}-2(v u v)_{x}
\end{array}\right. \\
\left\{\begin{array}{l}
u_{t}=u_{x x}+2 u_{x} v u, \\
v_{t}=-v_{x x}+2 v u v_{x},
\end{array}\right.
\end{gathered}
$$


The first system is a noncommutative generalization of the derivative nonlinear Schrödinger equation. The second system is interesting, since it gives another example of an integrable equation not associated with a Jordan algebra. The corresponding third order symmetry is

$$
\left\{\begin{array}{l}
u_{t}=u_{x x x}+3 u_{x x} v u+3 u_{x} v u_{x}+3 u_{x} v u v u \\
v_{t}=v_{x x x}-3 v u v_{x x}-3 v_{x} u v_{x}+3 v u v u v_{x}
\end{array}\right.
$$

\section{Matrix Painlevé Equations.}

It is well known that the symmetry reductions of integrable systems are ordinary differential equations of Painlevé type, $[\mathbf{2}],[\mathbf{3 0}]$. Thus, we can seek symmetry reductions of our noncommutative integrable equations, leading to associative algebra-valued counterparts of the classical Painlevé transcendents P-I, .., P-VI, [23].

First of all, the first Painleve transcendent P-I appears as a symmetry reduction of the matrix KdV equation (1.3). Namely, the classical Galilean symmetry

$$
\frac{\partial}{\partial t}-t \frac{\partial}{\partial x}+\frac{1}{6} \frac{\partial}{\partial u}
$$

gives us the group-invariant solution

$$
u=\frac{1}{6} t \mathbf{e}+v\left(x+\frac{1}{2} t^{2}\right)
$$

where $\mathbf{e}$ is the identity (or any other constant) element of the algebra, and $v$ satisfies the matrix ordinary differential equation

$$
v^{\prime \prime \prime}+3 v v^{\prime}+3 v^{\prime} v=\frac{1}{6} \mathbf{e}
$$

By integrating we obtain the first matrix Painlevé equation

$$
v^{\prime \prime}+3 v^{2}=\frac{1}{6} z \mathbf{e}+a
$$

with arbitrary constant matrix a. Of course, we can use the scaling to normalize the constants 3 and $\frac{1}{6}$. By a suitable shift of $z$ and a conjugation of the form $v \mapsto m v m^{-1}$, we can make $a$ into a matrix having zero trace in Jordan canonical form. Note that the Painlevé equation (5.1) admits a symmetric reduction $v^{*}=v$.

The two matrix mKdV equations lead to two different matrix version of the second Painlevé equation P-II. Namely, the simple scaling gives us the substitution

$$
u=t^{-1 / 3} v\left(x t^{-1 / 3}\right)
$$

Starting with the standard matrix $\operatorname{mKdV}(1.4)$, we obtain

$$
v^{\prime \prime \prime}+3 v^{2} v^{\prime}+3 v^{\prime} v^{2}+\frac{1}{3} v+\frac{1}{3} z v^{\prime}=0 .
$$

The second $\mathrm{mKdV}(1.4)$ (and the same reduction) gives rise to different third order ODE

$$
v^{\prime \prime \prime}+3 v v^{\prime \prime}-3 v^{\prime \prime} v-6 v v^{\prime} v+\frac{1}{3} v+\frac{1}{3} z v^{\prime}=0
$$


In contrast with the scalar case, we are unable to lower the order of either version of the second Painlevé transcendent, which remain third order equations.

In the scalar case the fourth Painleve equation P-IV is equivalent to the following system of Riccati type:

$$
\begin{aligned}
y^{\prime} & =c_{1}-\frac{1}{2} z y-y^{2}-2 y w \\
w^{\prime} & =c_{2}+\frac{1}{2} z w+w^{2}+2 y w,
\end{aligned}
$$

where $c_{1}, c_{2}$ are arbitrary constants. The system (5.4) can be most simply obtained from the commutative nonlinear Schrödinger type system (4.10) via the similarity reduction $u=t^{-1 / 2} y(z), v=t^{-1 / 2} w(z)$, with $z=t^{-1 / 2} x$. There are two different noncommutative counterparts to this equation, leading to two noncommutative versions of P-IV. Performing the same similarity reduction on (4.18) leads to

$$
\begin{aligned}
& y^{\prime \prime}+2 y y^{\prime}+\left(w y+\frac{1}{2} z y\right)^{\prime}+2 y w y-2 w y^{2}=0, \\
& w^{\prime \prime}-2 w^{\prime} w-\left(w y+\frac{1}{2} z w\right)^{\prime}+2 w y w-2 w^{2} y=0,
\end{aligned}
$$

whereas (4.19) yields the alternative system

$$
\begin{aligned}
& y^{\prime \prime}+2 y y^{\prime}+\left(y w+\frac{1}{2} z y\right)^{\prime}+2 y^{2} w-2 y w y=0, \\
& w^{\prime \prime}-2 w w^{\prime}-\left(w y+\frac{1}{2} z w\right)^{\prime}-2 w y w+2 w^{2} y=0 .
\end{aligned}
$$

Unlike the commutative version, neither of these can be reduced to a first order system.

The classical matrix chiral model

$$
u_{x y}=\left\{u_{x}, u^{-1}, u_{y}\right\}=\frac{1}{2}\left(u_{x} u^{-1} u_{y}+u_{y} u^{-1} u_{x}\right)
$$

is one of the most important integrable matrix equations. In $[45],[46],[47]$, this equation was generalized to the case of arbitrary Jordan triple system. The general scaling symmetry reduction of (5.7) leads to the substitution

$$
u=x^{p} y^{q} v\left(x^{\alpha} y^{\beta}\right), \quad \alpha \beta \neq 0 .
$$

Without loss of generality we can put $q=0$, but the resulting reduced matrix ordinary differential equation does not depend on $p, q, \alpha, \beta$ and is as follows:

$$
v_{z z}+\frac{v_{z}}{z}=v_{z} v^{-1} v_{z}, \quad z=x^{\alpha} y^{\beta}
$$

In the standard case, this equation is a special case of P-III.

It would be interesting to investigate the analytical properties, Bäcklund transformations and the corresponding isomonodromic problems for these matrix Painlevé equations. We do not know if there exist other matrix Painlevé equations (for instance P-VI). It seems possible to transfer our "associative approach" from symmetry analysis to the Painlevé analysis. This could be allow one to classify matrix ordinary differential equations of Painlevé type. For example, recently Balandin and the second author, [6], have shown that the matrix P-II equation

$$
v^{\prime \prime}=2 v^{3}+z v+\alpha \mathbf{e}
$$

where $u(z)$ is an unknown $n \times n$-matrix, $\mathbf{e}$ is the unit matrix, $\alpha$ is a scalar parameter, although it does not arise as a reduction of either of the matrix $m K d V$ equations, passes through the Painlevé-Kovalevskaya test. 


\section{Hamiltonian structures.}

A scalar evolution equation (3.1) is said to be Hamiltonian if it can be written in the form

$$
u_{t}=\mathcal{D} \delta \mathcal{H}
$$

Here $\mathcal{H}=\int H\left(u, u_{x}, \ldots\right) d x$ is the Hamiltonian functional, $\delta$ is the variational derivative, and the Hamiltonian operator $\mathcal{D}$ determines a Poisson bracket

$$
\{\mathcal{F}, \mathcal{H}\}=\int \delta \mathcal{F} \cdot \mathcal{D} \delta \mathcal{H} d x
$$

on the space of functionals. The Hamiltonian operator $\mathcal{D}$ is required to be a skew-adjoint differential operator, $\mathcal{D}^{*}=-\mathcal{D}$, in order that the Poisson bracket (6.2) be skew-symmetric, $\{\mathcal{F}, \mathcal{H}\}=-\{\mathcal{H}, \mathcal{F}\}$. In addition, we require that (6.2) satisfy the Jacobi identity

$$
\{\mathcal{F},\{\mathcal{G}, \mathcal{H}\}\}+\{\mathcal{G},\{\mathcal{H}, \mathcal{F}\}\}+\{\mathcal{H},\{\mathcal{F}, \mathcal{G}\}\}=0
$$

Any skew-adjoint operator which does not explicitly depend on the field variable $u$ automatically satisfies the Jacobi identity. For field dependent operators, (6.3) is a nontrivial condition which we discuss in more detail below. We refer the reader to [38; Chapter 7], for details on the general theory of (commutative) Hamiltonian systems.

Generalizing the calculus of Poisson brackets and variational derivatives to the noncommutative case is relatively straightforward. The basic functionals must still be scalarvalued, and thus must be expressed as integrals of certain trace forms. For example, the functional

$$
\mathcal{H}_{1}=\int \operatorname{tr}\left(-\frac{1}{2} u_{x}^{2}+u^{3}\right) d x
$$

turns out to be a conservation law for the non-commutative Korteweg-deVries equation (1.3). We can compute its variational derivative as follows

$$
\begin{aligned}
\left\langle\delta \mathcal{H}_{1} ; v\right\rangle & =\left.\frac{d}{d t} \mathcal{H}[u+t v]\right|_{t=0}=\int \operatorname{tr}\left(-\frac{1}{2} u_{x} v_{x}-\frac{1}{2} v_{x} u_{x}+v u^{2}+u v u+u^{2} v\right) d x \\
& =\int \operatorname{tr}\left(-u_{x} v_{x}+3 u^{2} v\right) d x=\int \operatorname{tr}\left[\left(u_{x x}+3 u^{2}\right) v\right] d x .
\end{aligned}
$$

Here we are using the fundamental property (2.5) of the trace operation as well as integration by parts. Therefore, the variational derivative of the functional (6.4) is

$$
\delta \mathcal{H}_{1}=u_{x x}+3 u^{2} .
$$

The associative algebra-valued Korteweg-deVries equation (1.3) can thus be written in the Hamiltonian form

$$
u_{t}=D_{x} \delta \mathcal{H} .
$$

The total derivative operator $D_{x}$, being skew-adjoint and independent of the field variable, is automatically Hamiltonian. 
For general skew-adjoint differential operators, the simplest method for verifying the Jacobi identity (6.3) is based on the calculus of "functional multi-vectors" developed in [38; Chapter 7]. We assume that the reader is familiar with the method for the commutative case as explained there. The non-commutative version is very similar; in the one component case, one introduces the basic uni-vectors $\theta_{k} \equiv D_{x}^{k} \theta$, which are, in a certain sense, "duals" to the one-forms $d u_{k}=D_{x}^{k} d u$. The main difference is that, since ordinary multiplication is no longer commutative, the wedge product of such multi-vectors is no longer skew symmetric. Thus, in order to avoid confusion, we shall drop the explicit wedge product symbol entirely, denoting $\theta \wedge \theta_{x}$, say, by just concatenation $\theta \theta_{x}$. The only identity that is retained in the case of an associative algebra is the skew analogue of the trace formula (2.5), which requires

$$
\operatorname{tr} \xi \eta=(-1)^{m n} \operatorname{tr} \eta \xi,
$$

for any $m$-vector $\xi$ and $n$-vector $\eta$. An associative algebra-valued functional multi-vector has the form $\Theta=\int \operatorname{tr} \xi d x$, where $\xi$ is a multi-vector built on the underlying associative algebra. We note that, besides the trace identity (6.5), one can also integrate functional multi-vectors by parts:

$$
\int \operatorname{tr}\left(\xi D_{x} \eta\right) d x=-\int \operatorname{tr}\left(\left(D_{x} \xi\right) \eta\right) d x=-\int \operatorname{tr}\left(\eta D_{x} \xi\right) d x .
$$

The most important functional multi-vector is the bivector

$$
\Theta=\int \operatorname{tr}(\theta \mathcal{D} \theta) d x
$$

associated with a skew-adjoint linear operator $\mathcal{D}$. The operator $\mathcal{D}$ defines a noncommutative Poisson bracket

$$
\{\mathcal{F}, \mathcal{H}\}=\int \operatorname{tr}[\delta \mathcal{F} \cdot \mathcal{D} \delta \mathcal{H}] d x,
$$

if and only if the bivector $\Theta$ satisfies the quadratic bracket condition

$$
[\Theta, \Theta]=2 \mathbf{v}_{\mathcal{D} \theta}(\Theta)=0
$$

Here $[\cdot, \cdot]$ denotes the noncommutative Schouten bracket, cf. [37], between functional multi-vectors. The functional trivector (6.9) can be effectively computed via the noncommutative version of a standard formula based on the formal evolutionary vector field $\mathbf{v}_{\mathcal{D} \theta}$ which has multi-vector characteristic $\mathcal{D} \theta$. In other words, we replace $K$ in (3.2) by the uni-vector $\mathcal{D} \theta$, and allow $\mathbf{v}_{\mathcal{D} \theta}$ to act as an anti-derivation on the space of multi-vectors, meaning that

$$
\mathbf{v}_{\mathcal{D} \theta}(\xi \eta)=\mathbf{v}_{\mathcal{D} \theta}(\xi) \eta+(-1)^{m} \xi \mathbf{v}_{\mathcal{D} \theta}(\eta)
$$

whenever $\xi$ is an $m$-vector. In other words, $\mathbf{v}_{\mathcal{D} \theta}$ picks up a minus sign each time it "moves past" another uni-vector. See $[\mathbf{3 8}]$ for details, which are straightforwardly adapted here to the non-commutative case. 
Two Hamiltonian operators $\mathcal{D}$ and $\mathcal{E}$ is said to form a Hamiltonian pair provided any linear combination $a \mathcal{D}+b \mathcal{E}, a, b \in \mathbb{R}$, is also Hamiltonian. This is equivalent to requiring that $\mathcal{D}$ and $\mathcal{E}$ are individually Hamiltonian, meaning that their associated bivectors

$$
\Theta=\int \operatorname{tr}(\theta \mathcal{D} \theta) d x, \quad \Xi=\int \operatorname{tr}(\theta \mathcal{E} \theta) d x
$$

satisfy the bracket condition (6.9). Moreover, they must satisfy a compatibility condition that the Schouten bracket between their associated bivectors vanishes, leading to the complete system of bracket conditions

$$
[\Theta, \Theta]=0, \quad[\Theta, \Xi]=0, \quad[\Xi, \Xi]=0,
$$

Magri's theorem, $[\mathbf{2 8}],[\mathbf{3 8}]$, demonstrates the integrability of any biHamiltonian system

$$
u_{t}=\mathcal{D} \delta \mathcal{H}_{1}=\mathcal{E} \delta \mathcal{H}_{0}
$$

Indeed, the operator

$$
\mathcal{R}=\mathcal{E} \cdot \mathcal{D}^{-1},
$$

forms a recursion operator for the system (6.12), leading to the hierarchy of commuting higher order flows

$$
u_{t}=\mathcal{D} \delta \mathcal{H}_{n+1}=\mathcal{E} \delta \mathcal{H}_{n},
$$

associated with the hierarchy $\mathcal{H}_{n}$ of higher order conservation laws. The main technical issue is whether the higher order flows generated by recursion are local.

We now discuss what is known concerning the Hamiltonian and biHamiltonian structures of noncommutative integrable systems.

Theorem 6.1. The noncommutative Korteweg-deVries equation (1.3) is a biHamiltonian system for the compatible Hamiltonian pair

$$
\mathcal{D}=D_{x}, \quad \mathcal{E}=D_{x}^{3}+A_{u} D_{x}+D_{x} A_{u}+C_{u} D_{x}^{-1} C_{u} .
$$

Here $A_{u}$ and $C_{u}$ are the anti-commutator and commutator maps as in (2.3).

Proof:

Note that

$$
\mathcal{D} v=v_{x}, \quad \mathcal{E} v=v_{x x x}+4\left\{u, v_{x}\right\}+2\left\{u_{x}, v\right\}+\left[D^{-1}[v, u], u\right] .
$$

Therefore, it suffices to prove that $\mathcal{E}$ is Hamiltonian, since the linear combination $\mathcal{E}+c \mathcal{D}$ can be simply obtained from $\mathcal{E}$ via translation $u \mapsto u+c$. We write $\mathcal{E}=\mathcal{E}_{0}+\mathcal{E}_{1}+\mathcal{E}_{2}$, where

$$
\mathcal{E}_{0}=D_{x}^{3}, \quad \mathcal{E}_{1}=2 A_{u} D_{x}+A_{u_{x}}, \quad \mathcal{E}_{2}=C_{u} D_{x}^{-1} C_{u} .
$$


Note that the subscript on the $\mathcal{E}^{\prime}$ s denotes their degrees (weights) under scaling in $u$. We similarly decompose the associated functional bivector: $\Theta=\Theta_{0}+\Theta_{1}+\Theta_{2}$. Let us introduce the noncommutative uni-vectors

$$
\lambda=D_{x}^{-1}(u \theta), \quad \rho=D_{x}^{-1}(\theta u) .
$$

Then using integration by parts (6.6) and the trace identity (6.5), we find the following simplified formulae:

$$
\begin{aligned}
& \Theta_{0}=\int \operatorname{tr}\left[\theta \theta_{x x x}\right] d x, \\
& \Theta_{1}=2 \int \operatorname{tr}\left[u \theta \theta \theta_{x}-u \theta_{x} \theta\right] d x=2 \int \operatorname{tr}\left[\left(\lambda_{x}+\rho_{x}\right) \theta_{x}\right] d x, \\
& \Theta_{2}=\int \operatorname{tr}\left[(\lambda-\rho)\left(\lambda_{x}-\rho_{x}\right)\right] d x .
\end{aligned}
$$

Since the Schouten bracket condition (6.9) is quadratic in $\Theta$, we can split the trivector $\Upsilon=[\Theta, \Theta]$ into homogeneous components $\Upsilon=\Upsilon_{0}+\Upsilon_{1}+\Upsilon_{2}+\Upsilon_{3}$, where

$$
\begin{array}{ll}
\Upsilon_{0}=\mathbf{v}_{0}\left(\Theta_{1}\right), & \Upsilon_{2}=\mathbf{v}_{1}\left(\Theta_{2}\right)+\mathbf{v}_{2}\left(\Theta_{1}\right), \\
\Upsilon_{1}=\mathbf{v}_{0}\left(\Theta_{2}\right)+\mathbf{v}_{1}\left(\Theta_{1}\right), & \Upsilon_{3}=\mathbf{v}_{2}\left(\Theta_{2}\right) .
\end{array}
$$

Here $\mathbf{v}_{k}=\mathbf{v}_{\mathcal{E}_{k} \theta}$, and we are using the fact that $\mathbf{v}_{k}\left(\Theta_{0}\right)=0$ since $\mathcal{E}_{0}$ is a constant coefficient operator. When evaluating (6.20), it is important to remember the anti-derivational rules (6.10) for the formal vector fields $\mathbf{v}_{k}=\mathbf{v}_{\mathcal{E}_{k} \theta}$. In particular,

$$
\mathbf{v}_{0}\left(\lambda_{x}\right)=\theta_{x x x} \theta, \quad \text { whereas } \quad \mathbf{v}_{0}\left(\rho_{x}\right)=-\theta \theta_{x x x} .
$$

Similarly, we find

$$
\begin{array}{ll}
\mathbf{v}_{1}\left(\lambda_{x}\right)=2 u \theta_{x} \theta+2 \theta_{x} \lambda_{x}+u_{x} \theta \theta+\theta u_{x} \theta, & \mathbf{v}_{2}\left(\lambda_{x}\right)=(\rho-\lambda) \lambda_{x}-u(\rho-\lambda) \theta, \\
\mathbf{v}_{1}\left(\rho_{x}\right)=-2 \theta \theta_{x} u-2 \rho_{x} \theta_{x}-\theta u_{x} \theta-\theta \theta u_{x}, & \mathbf{v}_{2}\left(\rho_{x}\right)=\rho_{x}(\rho-\lambda)-\theta(\rho-\lambda) u .
\end{array}
$$

We can now compute the relevant brackets. First, applying (6.21) and integrating the result by parts, we find

$$
\begin{aligned}
\mathbf{v}_{0}\left(\Theta_{1}\right) & =\int \operatorname{tr}\left[\mathbf{v}_{0}\left(\lambda_{x}\right)+\mathbf{v}_{0}\left(\rho_{x}\right) \theta_{x}\right] d x=\int \operatorname{tr}\left[\theta_{x x x} \theta \theta_{x}-\theta_{x x x} \theta_{x} \theta\right] d x \\
& =\int \operatorname{tr}\left[-\theta_{x x} \theta_{x} \theta_{x}-\theta_{x x} \theta \theta_{x x}+\theta_{x x} \theta_{x x} \theta+\theta_{x x} \theta_{x} \theta_{x}\right] d x=0,
\end{aligned}
$$

the final equality relying on (2.5). Similarly,

$$
\begin{aligned}
\mathbf{v}_{2}\left(\Theta_{2}\right) & =\int \operatorname{tr}\left[\mathbf{v}_{2}\left(\lambda_{x}\right)-\mathbf{v}_{2}\left(\rho_{x}\right)(\rho-\lambda)\right] d x \\
& =\int \operatorname{tr}\left[\left(\lambda_{x}-\rho_{x}\right)(\lambda \lambda-\lambda \rho-\rho \lambda+\rho \rho)\right] d x=0 .
\end{aligned}
$$

The last equality follows by noting that the various terms combine to form total derivatives and hence integrate to zero; for example, using (6.5),

$$
D_{x} \operatorname{tr} \lambda \lambda \lambda=\operatorname{tr}\left(\lambda_{x} \lambda \lambda+\lambda \lambda_{x} \lambda+\lambda \lambda \lambda_{x}\right)=3 \operatorname{tr} \lambda_{x} \lambda \lambda
$$

and hence $\int \operatorname{tr}\left(\lambda_{x} \lambda \lambda\right) d x=0$. The verification that $\Upsilon_{1}=0=\Upsilon_{2}$ is similar, although more tedious. 
Corollary 6.2. The operator

$$
\mathcal{R}=D_{x}^{2}+2 A_{u}+A_{u_{x}} D_{x}^{-1}+C_{u} D_{x}^{-1} C_{u} D_{x}^{-1}
$$

is a recursion operator for the non-commutative Korteweg-deVries equation (1.3).

Remark: The operator (6.23) is a special case of a formula due to Svinolupov, [49], for the recursion operator for multi-component Jordan systems. The proof that $\mathcal{E}$ satisfies the Jacobi identity would be almost impossible to do using the component form of the operator.

Remark: It is an open problem to prove that, as with the commutative KdV recursion operator, applying (6.23) recursively to the elementary symmetry $K_{0}=u_{x}$ produces a hierarchy of local, mutually commuting, higher order flows. We have verified this up to order 11 , but do not have a general proof.

There are several interesting points associated with this result, indicating that the noncommutative Hamiltonian theory is more complicated than the well-studied commutative theory. First, and most noticeable, is the fact that the operator $\mathcal{E}$ is non-local, requiring the formal integral operator $D_{x}^{-1}$. This is in contrast with the scalar Korteweg-deVries equation, whose second Hamiltonian structure is a local operator.

Second, and even more surprising, is that, except for the constant coefficient operator $D_{x}^{3}$, the other two homogeneous summands of the second Hamiltonian operator $\mathcal{E}$ are not individual Hamiltonian operators - only when they appear in the particular linear combination (6.15) does the operator define a genuine Poisson bracket! In particular, the combination $D_{x}^{3}+A_{u} D_{x}+D_{x} A_{u}$, which would appear to be a more natural noncommutative generalization of the second $\mathrm{KdV}$ Hamiltonian operator, is not Hamiltonian. Indeed, $\left[\Theta_{1}, \Theta_{1}\right]=\mathbf{v}_{1}\left(\Theta_{1}\right) \neq 0$, and hence $\mathcal{E}_{1}(v)=4\left\{u, v_{x}\right\}+2\left\{u_{x}, v\right\}$ does not define a Hamiltonian operator! This is remarkable, since this appears to be the direct analogue of the commutative Hamiltonian operator $2 u D_{x}+u_{x}$, which is the simplest Hamiltonian operator appearing in commutative Hamiltonian systems of "hydrodynamic type", [12], [13], $[\mathbf{1 4}],[\mathbf{3 9}]$, which are first order quasilinear systems of partial differential equations. The operator $2 u D_{x}+u_{x}$ defines the first of four known Hamiltonian structures for the inviscid Burgers' equation $u_{t}=u u_{x},[39]$. Our result indicates that the noncommutative theory of systems of hydrodynamic type is considerably more complicated than the commutative theory. However, this theory is clearly worth developing, since, in view of the connections between such Hamiltonian operators and Riemannian geometry in the commutative case, the resulting theory may help shed new light on how to construct a "noncommutative Riemannian geometry", cf. [8].

The general form for a first order Hamiltonian operator for the mKdV-type equations is

$$
\mathcal{D}=D_{x}+\lambda C_{u}+\mu C_{u} D_{x}^{-1} C_{u}
$$

In other words,

$$
\mathcal{D}(v)=v_{x}+\lambda[u, v]+\mu\left[u, D_{x}^{-1}[u, v]\right] .
$$

Therefore, the operators $D_{x}, C_{u}$, and $C_{u} D_{x}^{-1} C_{u}$, form a compatible Hamiltonian triple, although the latter is an immediate consequence of the compatibility of the first two 
operators. Interestingly, in contrast with the scalar modified Korteweg-deVries equation, whose first order Hamiltonian structure is the local operator $D_{x}$, the structure here is non-local, requiring the formal integral operator $D_{x}^{-1}$.

Theorem 6.3. For any scalar constants $\lambda, \mu$, the operator (6.24) is Hamiltonian.

The proof is similar to Theorem 6.1 and is omitted. Choosing $\lambda=0, \mu=1$, so

$$
\mathcal{D}_{1}=D_{x}+C_{u} D_{x}^{-1} C_{u},
$$

we find the that the first matrix $\mathrm{mKdV}$ equation (1.4) can be written in Hamiltonian form (6.1), with Hamiltonian

$$
\mathcal{H}_{1}=\int \operatorname{tr}\left(-\frac{1}{2} u_{x}^{2}+\frac{1}{2} u^{4}\right) d x
$$

Choosing $\lambda=3, \mu=2$, so

$$
\mathcal{D}_{2}=D_{x}+3 C_{u}+2 C_{u} D_{x}^{-1} C_{u}
$$

we find the that the second matrix $\mathrm{mKdV}$ equation (1.6) can be written in Hamiltonian form (6.1), with Hamiltonian

$$
\mathcal{H}_{2}=\int \operatorname{tr}\left(-\frac{1}{2} u_{x}^{2}-\frac{1}{2} u^{4}\right) d x
$$

For general $\lambda, \mu$, and Hamiltonian

$$
\mathcal{H}=\int \operatorname{tr}\left(-\frac{1}{2} u_{x}^{2}+\frac{1}{2} \varepsilon u^{4}\right) d x
$$

we obtain the family of matrix Hamiltonian equations

$$
u_{t}=u_{x x x}+\lambda\left[u, u_{x x}\right]+(\mu+\varepsilon)\left\{u^{2}, u_{x}\right\}+(\varepsilon-2 \mu) u u_{x} u .
$$

However, Theorem 3.6 indicates that not all of these are integrable. More specifically, only the first and second matrix mKdV equations (1.4), (1.6), admit a fifth order symmetry.

\section{Conclusions and Further Research.}

In this paper, we have initiated the systematic classification of integrable evolution equations whose field variables take values in an associative algebra. Complete classifications are found for equations of $\mathrm{KdV}$ type, where the only integrable noncommutative examples found so far are the noncommutative $\mathrm{KdV}$ and its higher order symmetries. A similar result holds for noncommutative generalizations of the $\mathrm{mKdV}$ equation. The classification of two-component systems generalizing the nonlinear Schrödinger equation and has been completed. In both the commutative and noncommutative cases, new examples of integrable systems were discovered. In some cases, commutative equations have one or two noncommutative counterparts, whereas some integrable commutative equations 
cannot be extended to the noncommutative regime. The computer packages used to effect these computations can be readily applied to other types of systems, although the required amount of computing power and memory increases rapidly, leaving many interesting cases unexplored. Explicit solutions, including solitons, as well as the possible linearizations and integration via a noncommutative inverse scattering method will be the subject of future research.

Symmetry reduction of the noncommutative integrable equations leads to a variety of associative algebra valued ordinary differential equations of Painlevé type, whose properties await a more detailed development. A systematic study of both symmetry reductions, and integrable reductions through specialization of the field variables, will be discussed in a future work.

Noncommutative Hamiltonian structures for some of the integrable equations were found, although the second Hamiltonian structure for the two noncommutative variants of the $\mathrm{mKdV}$ equation have not yet been determined. Another important problem is to prove that all the higher order symmetries of our noncommutative systems obtained through application of the nonlocal recursion operator are local equations.

Since the field variables in our systems take their value in an arbitrary associative algebra, our results can thus be equally well applied to algebras of operators over Hilbert spaces and other infinite dimensional spaces. This leads one to speculate on a possible nonlinear quantum mechanical evolution, governed by a operator evolution equation. Our approach to the classification of nonabelian equations can therefore be regarded as a constructive procedure of "nonlinear" quantization that can be applied to classical integrable systems. The effect of such evolutions on such basic physical assumptions as the superposition principle remains to be explored.

Acknowledgments: We would like to thank the Ordway endowment for supporting V. Sokolov's visit to Minnesota, during which this project was initiated. We would also like to thank V.E. Adler, I.Z. Golubchik and R.I. Yamilov for helpful discussions. 


\section{References}

[1] Ablowitz, M.J., and Clarkson, P.A., Solitons, Nonlinear Evolution Equations and the Inverse Scattering Transform, L.M.S. Lecture Notes in Mathematics, Vol. 149, Cambridge University Press, Cambridge, 1991.

[2] Ablowitz, M.J., Ramani, A., and Segur, H., A connection between nonlinear evolution equations and ordinary differential equations of $P$-type, J. Math. Phys. 21 (1980), 715-721.

[3] Antonowicz, M., and Fordy, A.P., Coupled KdV equations with multi-Hamiltonian structures, Physica D 28 (1987), 345-357.

[4] Athorne, C., and Fordy, A.P., Generalised KdV and MKdV equations associated with symmetric spaces, J. Phys. A 20 (1987), 1377-1386.

[5] Bakirov, I.M., On the symmetries of some system of evolution equations, preprint, 1991.

[6] Balandin, S.P., and Sokolov, V.V., On the Painlevé test for nonabelian equations, preprint, Ufa, 1996.

[7] Chen, J.Q., Group Representation Theory for Physicists, World Scientific, Singapore, 1989.

[8] Connes, A., Noncommutative Geometry, Academic Press, San Diego, 1994.

[9] Crumeyrolle, A., Orthogonal and Symplectic Clifford Algebras, Kluwer, Boston, 1990.

[10] Curtis, C.W., and Reiner, I., Representation Theory of Finite Groups and Associative Algebras, Interscience, New York, 1962.

[11] Drinfeld, V.G., and Sokolov, V.V., Lie algebras and equations of Korteweg-de Vries type, J. Soviet Math. 30 (1985), 1975-2036.

[12] Dubrovin, B.A., and Novikov, S.P., Hamiltonian formalism of one-dimensional systems of hydrodynamic type and the Bogolyubov-Whitham averaging method, Sov. Math. Dokl. 27 (1983), 665-669.

[13] Dubrovin, B.A., and Novikov, S.P., On Poisson brackets of hydrodynamic type, Sov. Math. Dokl. 30 (1984), 651-654.

[14] Dubrovin, B.A., and Novikov, S.P., Hydrodynamics of weakly deformed soliton lattices. Differential geometry and Hamiltonian theory, Russian Math. Surveys 44:6 (1989), 35-124.

[15] Ferapontov, E.V., Differential geometry of nonlocal Hamiltonian operators of hydrodynamic type, Func. Anal. Appl. 25 (1991), 195-204.

[16] Fokas, A.S., A symmetry approach to exactly solvable evolution equations, J. Math. Phys. 2 (1980), 1318-1325.

[17] Fordy, A.P., Derivative nonlinear Schrödinger equations and Hermitian symmetric spaces, J. Phys. A 17 (1984), 1235-1245.

[18] Fordy, A.P., and Kulish, P.P., Nonlinear Schrödinger equations and simple Lie algebras, Commun. Math. Phys. 89 (1983), 427-443.

[19] Gürses, M., and Karasu, A., Degenerate Svinolupov KdV systems, Phys. Lett. A 214 (1996), 21-26. 
[20] Habibullin, I.T., Sokolov, V.V., and Yamilov, R.I., Multi-component integrable systems and nonassociative structures, in: Nonlinear Physics: Theory and Experiment, World Scientific, Singapore, 1996, pp. 139-168.

[21] Ibragimov, N.H., and Shabat, A.B., Evolutionary equations with nontrivial Lie-Bäcklund group, Func. Anal. Appl. 14 (1980), 19-28.

[22] Ibragimov, N.H., and Shabat, A.B., Infinite Lie-Bäcklund algebras, Func. Anal. Appl. 14 (1980), 313-315.

[23] Ince, E.L., Ordinary Differential Equations, Dover, New York, 1956.

[24] Jacobson, N., Structure and Representations of Jordan Algebras, American Math. Soc. Colloquium Publ., vol. 39, Providence, R.I., 1968.

[25] Kaup, D.J., On the inverse scattering problem for cubic eigenvalue problems of the class $\psi_{x x x}+6 Q \psi_{x}+6 R \psi=\lambda \psi$, Stud. Appl. Math. 62 (1980), 189-216.

[26] Khalilov, F.A., and Khruslov, E.Ya., Matrix generalisation of the modified Korteweg-deVries equation, Inv. Prob. 6 (1990), 193-204.

[27] Kichenassamy, S., and Olver, P.J., Existence and non-existence of solitary wave solutions to higher order model evolution equations, SIAM J. Math. Anal. 23 (1992), 1141-1166.

[28] Magri, F., A simple model of the integrable Hamiltonian equation, J. Math. Phys. 19 (1978), 1156-1162.

[29] Marchenko, V.A., Nonlinear Equations and Operator Algebras, D. Reidel Pub. Co., Boston, 1988.

[30] McLeod, J.B., and Olver, P.J., The connection between partial differential equations soluble by inverse scattering and ordinary differential equations of Painlevé type, SIAM J. Math. Anal. 14 (1983), 488-506.

[31] Mikhailov, A.V., Shabat, A.B., and Sokolov, V.V., The symmetry approach to classification of integrable equations, in: What is Integrability?, V.E. Zakharov, ed., Springer Verlag, New York, 1990, pp. 115-184.

[32] Mikhailov, A.V., Shabat, A.B., and Yamilov, R.I., The symmetry approach to classification of nonlinear equations. Complete lists of integrable systems, Russian Math. Surveys 42:4 (1987), 1-63.

[33] Mikhailov, A.V., Shabat, A.B., and Yamilov, R.I., Extension of the module of invertible transformations. Classification of integrable systems, Comm. Math. Phys. 115 (1988), 1-19.

[34] Mokhov, O.I., Hamiltonian systems of hydrodynamic type and constant curvature metrics, Phys. Lett. A 166 (1992), 215-216.

[35] Mokhov, O.I., and Ferapontov, E.V., Non-local Hamiltonian operators of hydrodynamic type related to metrics of constant curvature, Russian Math. Surveys 45:3 (1990), 218-219.

[36] Olver, P.J., Evolution equations possessing infinitely many symmetries, J. Math. Phys. 18 (1977), 1212-1215.

[37] Olver, P.J., Hamiltonian perturbation theory and water waves, Contemp. Math. 28 (1984), 231-249. 
[38] Olver, P.J., Applications of Lie Groups to Differential Equations, Second Edition, Graduate Texts in Mathematics, vol. 107, Springer-Verlag, New York, 1993.

[39] Olver, P.J., and Nutku, Y., Hamiltonian structures for systems of hyperbolic conservation laws, J. Math. Phys. 29 (1988), 1610-1619.

[40] Porteous, I., Clifford Algebras, Cambridge Stud. Adv. Math., vol. 50, Cambridge University Press, Cambridge, 1995.

[41] Röhrl, H., Algebras and differential equations, Nagoya Math. J. 68 (1977), 59-122.

[42] Sawada, K., and Kotera, T., A method for finding $N$-soliton solutions of the K.d.V. equation and K.d.V.-like equation, Prog. Theor. Physics 51 (1974), 1355-1367.

[43] Sokolov, V.V., On the symmetries of evolution equations, Russian Math. Surveys 43:5 (1988), 165-204.

[44] Sokolov, V.V., and Shabat, A.B., Classification of integrable evolution equations, Soviet Math. Phys. Reviews C 4 (1984), 221-280.

[45] Sokolov, V.V., and Svinolupov, S.I., Vector-matrix generalizations of classical integrable equations, Theor. Math. Phys. 100 (1994), 959-962.

[46] Sokolov, V.V., and Svinolupov, S.I., Deformations of nonassociative algebras and integrable differential equations, Acta Appl. Math. 41 (1995), 323-339.

[47] Sokolov, V.V., and Svinolupov, S.I., Deformations of Jordan triple systems and integrable equations, Theor. Math. Phys. 108 (1996), 388-392.

[48] Svinolupov, S.I., On the analogues of the Burgers equation, Phys. Lett. A 135 (1989), 32-36.

[49] Svinolupov, S.I., Jordan algebras and generalized KdV equations, Theor. Math. Phys. 87 (1991), 611-620.

[50] Svinolupov, S.I., Generalized Schrödinger equations and Jordan pairs, Commun. Math. Phys. 143 (1992), 559-575.

[51] Svinolupov, S.I., Jordan algebras and integrable systems, Func. Anal. Appl. 27 (1993), 257-265.

[52] Walcher, S., Algebras and Differential Equations, Hadronic Press, Palm Harbor, Fla., 1991.

[53] Zhiber, A.V., and Shabat, A.B., Klein-Gordon equations with a nontrivial group, Sov. Phys. Dokl. 24 (1979), 607-609. 